\title{
An efficient shock-capturing central-type scheme for multidimensional relativistic flows
}

\section{Hydrodynamics}

\author{
L. Del Zanna and N. Bucciantini
}

Dipartimento di Astronomia e Scienza dello Spazio, Università degli Studi di Firenze, Largo E. Fermi 2, 50125 Firenze, Italy

Received 8 March 2002 / Accepted 22 May 2002

\begin{abstract}
Multidimensional shock-capturing numerical schemes for special relativistic hydrodynamics (RHD) are computationally more expensive than their correspondent Euler versions, due to the nonlinear relations between conservative and primitive variables and to the consequent complexity of the Jacobian matrices (needed for the spectral decomposition in most of the approximate Riemann solvers of common use). Here an efficient and easy-to-implement three-dimensional (3-D) shockcapturing scheme for ideal RHD is presented. Based on the algorithms developed by P. Londrillo \& L. Del Zanna (2000, ApJ, 530,508 ) for the non-relativistic magnetohydrodynamic (MHD) case, and having in mind its relativistic MHD extension (to appear in a forthcoming paper), the scheme uses high order (third) Convex Essentially Non-Oscillatory (CENO) finite difference interpolation routines and central-type averaged Riemann solvers, which do not make use of time-consuming characteristic decomposition. The scheme is very efficient and robust, and it gives results comparable to those obtained with more sophisticated algorithms, even in ultrarelativistic multidimensional test problems.
\end{abstract}

Key words. hydrodynamics - relativity - shock waves - methods: numerical

\section{Introduction}

Relativistic flows and shocks play an essential role in modern high energy astrophysics, both for the interpretation of various observed features and for the description of the physical processes they give rise to, as, for example, the acceleration of highly energetic particles. Among the various astrophysical objects in which relativistic flows have been invoked to explain the observed properties, the best studied are probably:

1. Active galactic nuclei (AGNs), for which the presence of relativistic bulk motions (up to Lorentz factors of order 10) was soon suggested (Rees 1967). Associated with AGNs are often highly collimated relativistic jets (Begelman et al. 1984; and, for a review, Ferrari 1998), seen as apparent super-luminal lobes in some radio-loud sources and whose shock fronts are among the most promising candidates for high-energy particle acceleration. Mildly relativistic jets appear to be also associated with galactic Xray compact sources (generally called microquasars, see Mirabel \& Rodriguez 1999, for a review).

2. Gamma-ray bursts (GRBs), supposed to be originated during the collapse of the iron core of massive stars and the subsequent fireball explosion (Piran 1999; Kobayashi et al. 1999), which give rise to an expanding blast wave of

Send offprint requests to: L. Del Zanna,

e-mail: 1dz@arcetri.astro.it pairs and hadrons with typical Lorentz factors of $10^{2}-10^{3}$ (Meszaros \& Rees 1992), whose kinetic energy is then believed to be converted into gamma rays via cyclotron radiation and/or inverse Compton scattering.

3. Pulsar wind nebulae, assumed to be bubbles of relativistic particles and magnetic fields emitted by a pulsar as a relativistic wind with Lorentz factor ranging from $10^{4}$ to $10^{7}$, depending on the model of pair production in the pulsar magnetosphere (Michel \& Li 1999). The wind region may be confined by a termination shock, generated by the interaction with outer supernova matter, as is the case for the synchrotron emitting nebulae called plerions (Rees \& Gunn 1974; Kennel \& Coroniti 1984); or, if the pulsar is moving with supersonic speed through the interstellar medium, by the resulting ram pressure, in this case giving rise to a bow-shock pulsar wind nebula that may be detected in $\mathrm{H} \alpha$ (Bucciantini \& Bandiera 2001).

As we can see from these few but important examples, there is a strong interest in the astrophysical community in the development of computational codes for the numerical modeling and simulation of relativistic flows.

Over the last decade, high resolution shock-capturing methods of the Godunov type, successfully applied in classical fluid dynamics, have started to be employed for the case of relativistic hydrodynamics as well (Marquina et al. 1992; Schneider et al. 1993; Balsara 1994; Duncan \& Hughes 1994; 
Eulderink \& Mellema 1994; Font et al. 1994; Dolezal \& Wong 1995; Falle \& Komissarov 1996; Donat et al. 1998; Aloy et al. 1999). These schemes are characterized by the following main features: a conservative form of the discretized equations, in order to capture weak solutions and satisfy jump relations; a reconstruction phase, to recover variables at inter-cell locations where fluxes have to be computed; an upwind phase, in which an exact or approximate solution to the local Riemann problem is found. The simulated flows achieve high accuracy in smooth regions and, at the same time, shock profiles are stable and sharply defined. This is the reason for the great success of upwind differencing over central (or spectral) differencing. In the latter case, artificial viscosity must be introduced in order to damp the spurious oscillations that always form near discontinuities (Gibbs phenomena), thus leading to artificial heating and unwanted damping of physical waves.

When discontinuous solutions are of main interest, second order (both in time and space) total variation diminishing (TVD) schemes coupled with an accurate Riemann solver are probably the best choice, in terms of sharp resolution of discontinuities and overall stability. However, in the general case smooth wave-like or even turbulent fields appear together with discontinuous solutions, thus second order schemes are no longer able to resolve both features with enough precision. By relaxing the stringent TVD condition, a class of essentially non-oscillatory (ENO) higher-order schemes were first proposed by Harten et al. (1987), later modified in a more efficient implementation by Shu \& Osher (1989). The ENO philosophy is to use adaptive stencils to reconstruct variables and fluxes at cell interfaces. Thus, in smooth regions symmetric stencils will be used, whereas near discontinuities the stencil will shift to the left or to the right, selecting the smoother part of the flow and thus achieving the same high resolution (typically from third to fifth order in the case of weighted ENO schemes, see Jiang \& Shu 1996) everywhere, without the need of expensive adaptive mesh refinement (AMR) techniques. The price to pay is the presence, near shocks, of small oscillations of the order of the truncation error (Gibbs oscillations given by centered stencils that cross a discontinuity would be much higher, since they are proportional to the jump itself, independently on the resolution).

As for most TVD Godunov-type schemes, for ENO algorithms the local spectral decomposition in the building of numerical fluxes is also commonly adopted. It was already strongly advocated in the original paper, where a version of the ENO scheme with the (much) simpler component-wise reconstructions was found to fail, giving significant oscillations, in some Riemann problems. However, due to the high resolution of ENO methods, characteristic decomposition on every point of the interpolation stencil becomes prohibitive when moving to three-dimensional simulations, especially for relativistic flows where Jacobian matrices are more complex than in the Eulerian case. The same kind of problem has to be faced in magnetohydrodynamics (MHD, see Londrillo \& Del Zanna 2000 , from now on LD), because of the increasing number of variables, equations and eigenmodes. The worst possible case is obviously that of relativistic MHD, for which only second order 1-D (Balsara 2001) and 2-D (Komissarov 1999) Godunov schemes have been presented so far.

Following the scheme proposed in LD, in the present series of two papers a shock-capturing scheme that avoids the expensive characteristic decomposition, still retaining nonoscillatory properties, will be suggested, first for ideal relativistic hydrodynamics (RHD, in this paper) and then for ideal relativistic magnetohydrodynamics (RMHD, in the next paper of the series). Our method is based on the so-called central schemes, that extend the first-order local Lax-Friedrichs (LLF) flux splitting and assume an average over the Riemann fan at every cell interface (see Nessyahu \& Tadmor 1990, for the original paper and Kurganov et al. 2001, for the latest developments in this field). The basic properties of our method can be found in Liu \& Osher (1998), where a third order multidimensional central scheme was presented, with the following main features:

1. Point values rather than cell averages are used (that is to say finite differences instead of finite volumes, see Sect. 3 for details), thus removing the need of staggered grids (employed in previous central schemes) and making the extension to multiple dimensions a trivial task.

2. The semi-discrete form of the equations is solved, so that time integration can be achieved with any solver for ordinary differential equations (ODEs), for example TVD Runge-Kutta methods.

3. No characteristic decomposition and Riemann solvers are required: fluxes are reconstructed and derived componentwise, thus achieving a great simplicity in the programming and, above all, efficiency. The only spectral pieces of information needed are the local highest characteristic speeds (related to the Courant coefficient).

4. A new third order reconstruction algorithm is introduced and tested, called Convex-ENO (CENO), which has the fundamental property to stay as close as possible to the lower order TVD limited linear reconstruction (by using the minmod limiter in this case), thus reducing even to first order where needed, while retaining high accuracy in smooth region. This was recognized to be the key point of the surprising success of central schemes (Tadmor, cited in their acknowledgements), confirmed for the MHD case by LD.

Here, as in LD, we take advantage of all these positive features and we modify the original scheme by splitting the CENO reconstruction into two separate routines, so that reconstruction can be applied to primitive variables rather than to fluxes (the resulting scheme appears to be more robust, especially in the relativistic case). Moreover, different limiters (e.g. the monotonized centered) and solvers (the upwind HLL, from Harten, Lax, and van Leer) are introduced and tested in our framework.

We will show that our scheme, by providing an overall high resolution, is able to compensate for the larger smearing of discontinuities, especially contact discontinuities (due to the use of solvers based on just one or two characteristic speeds) and the results are comparable with those obtained by more sophisticated (but much more computationally expensive, especially 
in 3-D) relativistic codes. Concluding, notice that the proposed scheme can be applied unchanged to Euler HD too, where only the definition of conservative variables, fluxes and characteristic speeds are different.

\section{Ideal RHD equations}

The covariant equations in special relativistic hydrodynamics (RHD) are (e.g. Landau \& Lifshitz 1959):

$\partial_{\alpha}\left(\rho u^{\alpha}\right)=0$,

$\partial_{\alpha}\left(w u^{\alpha} u^{\beta}+p g^{\alpha \beta}\right)=0$,

where velocities are normalized against the speed of light $(c=$ 1) and Greek letters indicate four-vectors, while Latin indexes will be devoted to spatial 3-D vectors. The metric tensor defines space-time properties and here a Minkowski flat space with $g^{\alpha \beta}=\operatorname{diag}\{-1,1,1,1\}$ will be assumed throughout for simplicity, with coordinates $x^{\alpha}=\left(t, x^{j}\right)$. The physical quantities involved in the conservation laws are the rest mass density $\rho$, the kinetic pressure $p$, the relativistic enthalpy $w=e+p(e$ is the energy per unit volume, including rest mass energy) and the four-velocity $u^{\alpha}=\left(\gamma, \gamma v^{j}\right)$, where $\gamma=\left(1-v^{2}\right)^{-1 / 2}$ is the Lorentz factor. The system has to be closed with an equation of state $p=p(\rho, e)$, and here the relation for an ideal gas

$p=(\Gamma-1)(e-\rho) \Rightarrow e=\rho+p /(\Gamma-1)$

will be considered, where the adiabatic index should be taken as $\Gamma=5 / 3$ for the mildly relativistic case and as $\Gamma=4 / 3$ for the ultrarelativistic case $e \gg \rho$.

Godunov-type shock-capturing numerical methods developed for classical Euler equations can actually be applied to any multidimensional system of hyperbolic conservation laws of the form

$\frac{\partial \boldsymbol{u}}{\partial t}+\sum_{i=1}^{d} \frac{\partial \boldsymbol{f}^{i}(\boldsymbol{u})}{\partial x^{i}}=0$,

where $\boldsymbol{u}$ is the vector of conserved variables and $\boldsymbol{f}^{i}$ are their corresponding fluxes, along each direction ( $d$ is the number of spatial dimensions). Equations (1) and (2) are automatically cast in this form by just defining

$\boldsymbol{u}(\boldsymbol{v})=\left[\rho \gamma, w \gamma^{2} v^{j}, w \gamma^{2}-p\right]^{T}$,

$\boldsymbol{f}^{i}(\boldsymbol{v})=\left[\rho \gamma v^{i}, w \gamma^{2} v^{i} v^{j}+p \delta^{i j}, w \gamma^{2} v^{i}\right]^{T}$,

where $v=\left[\rho, v^{j}, p\right]^{T}$ are called primitive variables, and therefore the numerical techniques largely used for Euler HD equations can be applied to RHD too. The hyperbolic nature of Eq. (4) is guaranteed provided the local sound velocity $c_{\mathrm{s}}$ is sub-luminal, that is for causal equations of state (Anile 1989). For $\Gamma$-law gases where Eq. (3) holds, the relativistic sound speed is given by

$c_{\mathrm{s}}^{2}=\left(\frac{\partial p}{\partial e}\right)_{s}=\left(\frac{\partial e}{\partial p}\right)_{s}^{-1}=a^{2}\left(1+\frac{a^{2}}{\Gamma-1}\right)^{-1} \equiv \Gamma p / w$, which is obviously always less than unity, where $s \sim p \rho^{-\Gamma}$ is the specific entropy and $a^{2}=\Gamma p \rho^{-1}$ defines the classical sound speed.

In any numerical time advancing routine, primitive variables $\boldsymbol{v}$ have to be derived from the conservative ones at least once per time step, and if this is trivial for Euler equations it is not so in the RHD case, in which a numerical nonlinear rootfinding technique must be employed. If we define $W=w \gamma^{2}$ and

$\boldsymbol{u}=\left[D, Q^{j}, E\right]^{T}$,

where $D=\rho \gamma, Q^{j}=W v^{j}$, and $E=W-p$, the system to be inverted can be cast in the single equation for $\gamma$ :

$W(\gamma)^{2}\left(1-\gamma^{-2}\right)-Q^{2}=0$,

with $w=\rho+\Gamma_{1} p \Rightarrow W=D \gamma+\Gamma_{1} \gamma^{2} p$, here $\Gamma_{1}=\Gamma /(\Gamma-1)$, and $p=W-E$, so that

$W(\gamma)=\frac{E \Gamma_{1} \gamma^{2}-D \gamma}{\Gamma_{1} \gamma^{2}-1}$

Once the Lorentz factor is found numerically with the requested accuracy, the primitive variables are easily recovered thanks to the relations above.

\section{A novel ENO-based central scheme}

In this section ENO methods and their implementation for component-wise central schemes, together with our specific modifications, will be presented. For a general introduction and review of ENO methods for hyperbolic conservation laws see Shu (1997).

Consider a numerical discretization of Eq. (4), in the onedimensional case $d=1$ to begin with. Given an interval $[a, b]$, $N$ numerical cells $I_{i}=\left[x_{i-1 / 2}, x_{i+1 / 2}\right]$ of equal length $\Delta x=(b-$ $a) / N$ can be defined, with cell centers (grid nodes) given by

$x_{i}=a+(i-1 / 2) \Delta x ; i=1, \ldots, N$.

To an order $r$ of spatial accuracy, the numerical value of any quantity $v(x)$ (at a given time) will be denoted as $v_{i}=$ $v\left(x_{i}\right)+O\left((\Delta x)^{r}\right)$ at grid points (the so-called point values) or $v_{i \pm 1 / 2}=v\left(x_{i \pm 1 / 2}\right)+O\left((\Delta x)^{r}\right)$ at cell boundaries, where the order of accuracy refers only to smooth regions, in which the larger stencil can be used for interpolation. Moreover, cell averages are defined as

$\bar{v}_{i}=\frac{1}{\Delta x} \int_{x_{i-1 / 2}}^{x_{i+1 / 2}} v(x) \mathrm{d} x$,

and only for schemes up to second order, $r \leq 2$, they do coincide with point values $v_{i}$.

Most of the shock-capturing schemes evolve the cell averaged conserved quantities $\overline{\boldsymbol{u}}_{i}$ in time, as obtained by integrating Eq. (4) over the cell $I_{i}$. However, in the multidimensional case, the resulting numerical fluxes $\hat{f}_{i}$, that discretize the physical ones along one direction, have to be averaged along the transverse directions. This implies a truly multidimensional numerical interpolation for high order schemes, which is usually complex and computationally expensive. This approach is generally 
referred to as the finite volume approximation. Like in LD and in many other ENO schemes, from the works by Shu \& Osher (1988, 1989) onwards, we will adopt here finite difference approximations, based on point values. In the semi-discrete formalism (that is retaining continuous time dependency in the spatially discretized quantities), Eq. (4) becomes $(d=1)$ :

$\frac{\mathrm{d} \boldsymbol{u}_{i}}{\mathrm{~d} t}=-\frac{\hat{\boldsymbol{f}}_{i+1 / 2}-\hat{\boldsymbol{f}}_{i-1 / 2}}{\Delta x}$,

where $\hat{f}_{i \pm 1 / 2}$ are high-order approximations to the primitives of physical fluxes, that is to say that cell averages of the $\hat{f}(x)$ function must coincide with point values $f_{i}$ of the flux function $f(x)$, to the given accuracy $\left(\hat{f}_{i \pm 1 / 2} \equiv \boldsymbol{f}_{i \pm 1 / 2}\right.$ up to second order). The extension to the multidimensional case is now straightforward, since interpolations from cell centers to cell boundaries are made separately, dimension by dimension, and the other derivatives are just subtracted from the right hand side of Eq. (13) exactly in the same fashion as for the discretized $x$ derivative.

As specified by Shu \& Osher (1988), Eq. (13) must be integrated in time by using proper multi-level Runge-Kutta methods corresponding to the high order of spatial accuracy, so here we will always employ the optimal third order TVD algorithm:

$$
\begin{aligned}
& \boldsymbol{u}^{(1)}=\boldsymbol{u}^{n}+\Delta t \mathcal{L}\left[\boldsymbol{u}^{n}\right] \\
& \boldsymbol{u}^{(2)}=\frac{3}{4} \boldsymbol{u}^{n}+\frac{1}{4} \boldsymbol{u}^{(1)}+\frac{1}{4} \Delta t \mathcal{L}\left[\boldsymbol{u}^{(1)}\right] \\
& \boldsymbol{u}^{n+1}=\frac{1}{3} \boldsymbol{u}^{n}+\frac{2}{3} \boldsymbol{u}^{(2)}+\frac{2}{3} \Delta t \mathcal{L}\left[\boldsymbol{u}^{(2)}\right]
\end{aligned}
$$

where the superscript $n$ indicates the time stepping discretization, $\boldsymbol{u}^{(1)}$ and $\boldsymbol{u}^{(2)}$ refer to intermediate integration stages, and $\mathcal{L}[\boldsymbol{u}]$ is simply the right hand side of Eq. (13). The explicit time advancing scheme above is stable under the CFL (CourantFriedrichs-Lewy) condition $c<1$, where the Courant coefficient appears in the definition of the maximum time step allowed:

$$
\Delta t=\frac{c}{\max _{i}\left(\alpha^{i} / \Delta x^{i}\right)},
$$

with $\alpha^{i}$ being the largest speed of propagation of characteristic waves in the direction $i$. In the relativistic case, there is clearly a lower limit for the time step, given in Eq. (15) by simply taking $\alpha^{i}=1$ along all directions.

In order to complete the description of the scheme, the interpolation techniques and the approximate Riemann solver that defines the numerical inter-cell fluxes $\hat{\boldsymbol{f}}_{i+1 / 2}$ must be given. The following steps are taken, for every Runge-Kutta sub-cycle and for every direction in the multidimensional case:

1. Primitive variables are recovered from the conservative ones, according to the recipe already given in the previous section (this step is actually taken just once for each sub-cycle):

$$
\left\{\boldsymbol{u}_{i}\right\} \longrightarrow\left\{\boldsymbol{v}_{i}\right\} ; i=1, \ldots, N .
$$

2. Primitive variables are reconstructed at cell interfaces to give two states, called left and right inter-cell states:

$\left\{\boldsymbol{v}_{i}\right\} \longrightarrow\left\{\boldsymbol{v}_{i+1 / 2}^{\mathrm{L}}\right\},\left\{\boldsymbol{v}_{i+1 / 2}^{\mathrm{R}}\right\} ; i=0, \ldots, N$
The two reconstructions are based on polynomial interpolation over different sets of stencils, one centered on $x_{i}$ to approximate the left state $\boldsymbol{v}_{i+1 / 2}^{\mathrm{L}}$ and the other centered on $x_{i+1}$ to approximate the right state $\boldsymbol{v}_{i+1 / 2}^{\mathrm{R}}$. The interpolation is performed separately on each variable $v(x)$ according to a Rec routine based on the CENO (Convex ENO, Liu \& Osher 1998) technique, as described in Appendix A. Contrary to common ENO schemes, our method uses point values $v_{i}$ to yield point value reconstructed quantities $v_{i+1 / 2}$, instead of starting from cell averages $\bar{v}_{i}$. The order of the reconstruction is $r=3$ in smooth regions, but it reduces to linear reconstruction or even to first order (by using minmod-type limiters) near discontinuities. In this latter case $v_{i+1 / 2}^{\mathrm{L}}=v_{i}$ and $v_{i+1 / 2}^{\mathrm{R}}=v_{i+1}$, for each discontinuous field.

3. At each inter-cell point $x_{i+1 / 2}$ the local Riemann problem must be solved, in some approximate way. The most accurate solution would be given by an exact solver that computes the evolved state from $\boldsymbol{v}^{\mathrm{L}}$ and $\boldsymbol{v}^{\mathrm{R}}$ and then the correspondent flux, to be finally identified with $\boldsymbol{f}_{i+1 / 2}$. Another possibility is to define an average state $\tilde{\boldsymbol{v}}_{i+1 / 2}$ and to decompose fluxes according to a linearized problem with the Jacobian $\partial \boldsymbol{f} / \partial \boldsymbol{u}$ calculated there (Roe matrix approach). Our choice is to avoid spectral decomposition, which is computationally expensive, and to take an average over the local Riemann fan as in central-type schemes, already mentioned in the introduction. A simple two-speeds Riemann solver is the HLL one, first used in central schemes by Kurganov et al. (2001), that retains an upwind nature in the sense that coincides with $f^{\mathrm{L}}$ or $\boldsymbol{f}^{\mathrm{R}}$ if the Riemann fan does not cross the inter-cell itself (all the eigenvalues have the same sign). This may be written in the form

$f^{\mathrm{HLL}}=\frac{\alpha^{+} \boldsymbol{f}^{\mathrm{L}}+\alpha^{-} \boldsymbol{f}^{\mathrm{R}}-\alpha^{+} \alpha^{-}\left(\boldsymbol{u}^{\mathrm{R}}-\boldsymbol{u}^{\mathrm{L}}\right)}{\alpha^{+}+\alpha^{-}}$,

where all quantities are calculated from the reconstructed values of Eq. (17) by using Eqs. (5) and (6). Here the $\alpha^{ \pm}$ coefficients take into account the highest speeds at the two sides of the Riemann fan, which can be estimated from the maximum and minimum eigenvalue $\lambda^{ \pm}$of the Jacobians at the left and right states:

$\alpha^{ \pm}=\max \left\{0, \pm \lambda^{ \pm}\left(\boldsymbol{v}^{\mathrm{L}}\right), \pm \lambda^{ \pm}\left(\boldsymbol{v}^{\mathrm{R}}\right)\right\} \cdot$

For relativistic flows, the required eigenvalues are (after splitting the velocity along and perpendicular to the direction of spatial derivation, according to the relativistic rule for addition of velocity vectors):

$\lambda^{ \pm}=\frac{v_{\|}\left(1-c_{\mathrm{s}}^{2}\right) \pm c_{\mathrm{s}} \sqrt{\left(1-v^{2}\right)\left(1-v_{\|}^{2}-v_{\perp}^{2} c_{\mathrm{S}}^{2}\right)}}{1-v^{2} c_{\mathrm{s}}^{2}}$

that reduce simply to $\lambda^{ \pm}=\left(v \pm c_{\mathrm{s}}\right) /\left(1 \pm v c_{\mathrm{s}}\right)$ in the onedimensional case. Note that the maximum and minimum eigenvalues at the two reconstructed states are the only spectral pieces of information required. In this way shocks are handled correctly, whereas contact discontinuities and shear waves, corresponding to intermediate eigenvalues 
$\lambda=v_{\|}$, can appear somehow smeared, when compared with the results from proper Riemann solvers.

The simplest, smoothest, but also most dissipative numerical flux is the (local) Lax-Friedrichs one, given by

$\boldsymbol{f}^{\mathrm{LLF}}=\frac{1}{2}\left[\boldsymbol{f}^{\mathrm{L}}+\boldsymbol{f}^{\mathrm{R}}-\alpha\left(\boldsymbol{u}^{\mathrm{R}}-\boldsymbol{u}^{\mathrm{L}}\right)\right]$,

in which $\alpha=\max \left\{\alpha^{+}, \alpha^{-}\right\}$and therefore the averaging region is always symmetric with respect to $x_{i+1 / 2}$ (the LLF numerical flux is the prototype of central schemes). Note that the maximum of the set of values $\left\{\alpha_{i+1 / 2}\right\}$ yields the CFL-related $\alpha$ coefficient appearing in Eq. (15), and actually they coincide for the so-called global LF numerical flux. The calculation of eigenvalues may be avoided completely in a global LF scheme that uses $\alpha=1$ everywhere, although this is the most smearing case. Note that Eq. (18) reduces to Eq. (21) when $\alpha^{+}=\alpha^{-}=\alpha$, that is for symmetric Riemann fans in which $v_{\|}=0 \Rightarrow \lambda^{+}=-\lambda^{-}$.

4. From the point values of numerical fluxes, the approximations of their derivatives must be finally calculated (for lower than third order schemes this step can be avoided):

$\left\{\boldsymbol{f}_{i+1 / 2}\right\} \longrightarrow\left\{\hat{\boldsymbol{f}}_{i+1 / 2}\right\} ; i=0, \ldots, N$.

These are the numerical fluxes that actually enter Eq. (13). This last step usually does not appear in other ENO highorder schemes, since the reconstruction is made directly on fluxes and steps 2 and 4 are taken simultaneously (the numerical flux is calculated before reconstruction, using flux splitting methods). However, we have noticed that for central-type schemes like the one presented here, which avoid characteristic decomposition, the proposed approach is more robust and less oscillatory. This final interpolation is again performed separately on each variable with another CENO-based routine (Der, see Appendix A).

\section{Numerical results}

In this section the proposed scheme is validated against typical tests available in the current literature, separated here into one-dimensional tests, essentially shock-tube Riemann problems, and a few 2-D and 3-D experiments. All the simulations have been run on a single PC-Linux (AMD Athlon $1 \mathrm{GHz}$ CPU, 512Mb RAM, Pacific-Sierra Vast/f90 compiler, -O2 optimization and single precision calculations) in order to demonstrate that our scheme is not particularly demanding in terms of computational resources. In 3-D, with a grid of $100^{3}$ nodes, typical speeds are of about 2 mins per Runge-Kutta iteration (three internal sub-cycles for the third order scheme), so a simulation like the spherical explosion takes a few hours of computational time. However, the code is fully parallelized with Message Passing Interface (MPI) directives and has been successfully tested on a variety of supercomputers, like CrayT3E, IBM SP3/4 and Beowulf clusters.

In the following tests, we will always use the proposed third order CENO3 as a base scheme, unless specified otherwise. The approximate Riemann solvers employed are the HLL or the LLF, as described in the previous section, whereas

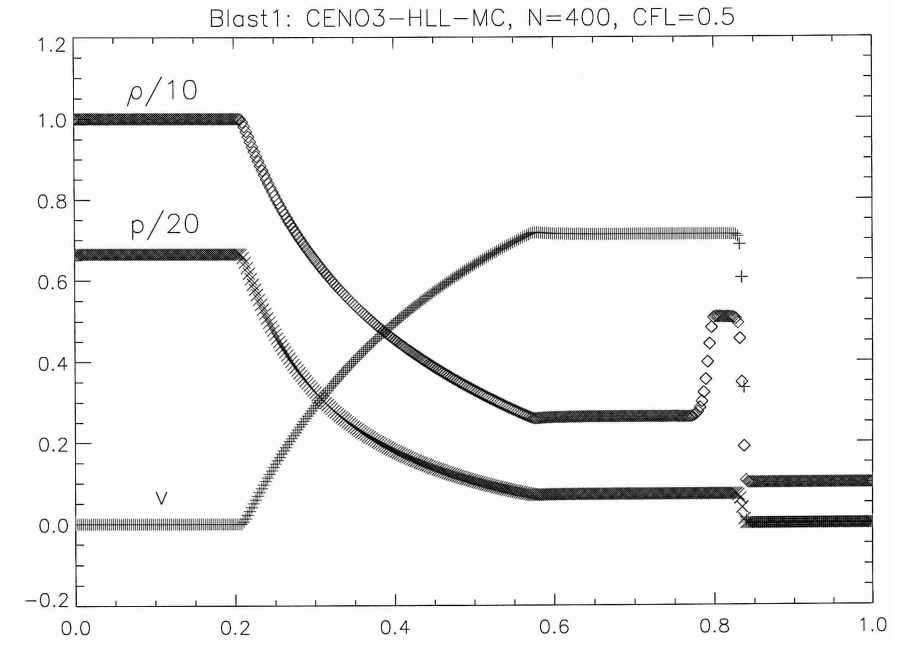

Fig. 1. The relativistic blast wave problem 1 for time $t=0.4$. The computed profiles of density (diamond), pressure (cross), and velocity (plus) are shown against distance. The base third order CENO3 scheme is employed with MC limiter and HLL solver. The computational grid is formed by $N=400$ equidistant cells and the Courant number is $c=0.5$.

the slope limiters used in the linear part of the reconstruction (see Appendix A) are the Monotonized Centered (MC) or the MinMod (MM). All the simulations presented here use the classical value $\Gamma=5 / 3$ for the adiabatic index.

\subsection{One-dimensional tests}

Shock-capturing numerical schemes, as in the classical hydrodynamical case, must be able to reproduce the discontinuous profiles involved in the so-called shock-tube problems. Given a unitary numerical 1-D pipe of $N$ grid points, two constant states $(\rho, v, p)$ are taken on the left $(0 \leq x \leq 0.5)$ and on the right $(0.5<x \leq 1)$ with respect of a diaphragm, placed initially at $x=0.5$ and then removed. Typical patterns seen in the subsequent evolution are shocks, contact discontinuities (characterized by density jumps not accompanied by discontinuities in normal velocity and pressure) and rarefaction waves. In the relativistic regime these features are qualitatively unchanged, since the structure of the characteristics is the same, but density jumps are not limited by any function of the adiabatic index and rarefaction waves do not yield straight profiles, due to the nonlinear Lorentz transformation formulae.

First we present two relativistic blast wave explosion problems, characterized by an initial static state with temperature and pressure much higher in the region on the left:

Blast wave 1: $\left\{\begin{array}{l}(\rho, v, p)^{\mathrm{L}}=(10,0,13.3), \\ (\rho, v, p)^{\mathrm{R}}=\left(1,0,10^{-6}\right),\end{array}\right.$
Blast wave 2: $\left\{\begin{array}{l}(\rho, v, p)^{\mathrm{L}}=(1,0,1000), \\ (\rho, v, p)^{\mathrm{R}}=(1,0,0.01),\end{array}\right.$

as in the Donat et al. (1998) paper. The first test is only mildly relativistic, while the second is more severe, with a shock speed corresponding to $\gamma \simeq 6$. In Figs. 1 and 2 the two tests are simulated with $N=400$ and Courant number $c=0.5$, with 


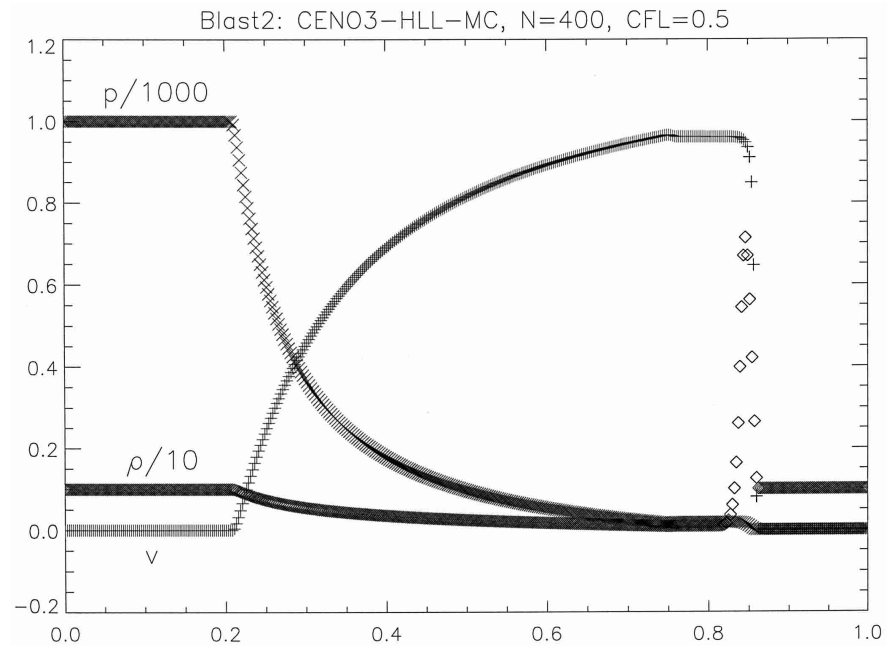

Fig. 2. The relativistic blast wave problem 2 for time $t=0.35$. The computed profiles of density (diamond), pressure (cross), and velocity (plus) are shown against distance. The base third order CENO3 scheme is employed with MC limiter and HLL solver. The computational grid is formed by $N=400$ equidistant cells and the Courant number is $c=0.5$. The exact value for the density peak is around 10.5 , our numerical result is around 7.3, similar to what is found by other third order more sophisticated schemes.

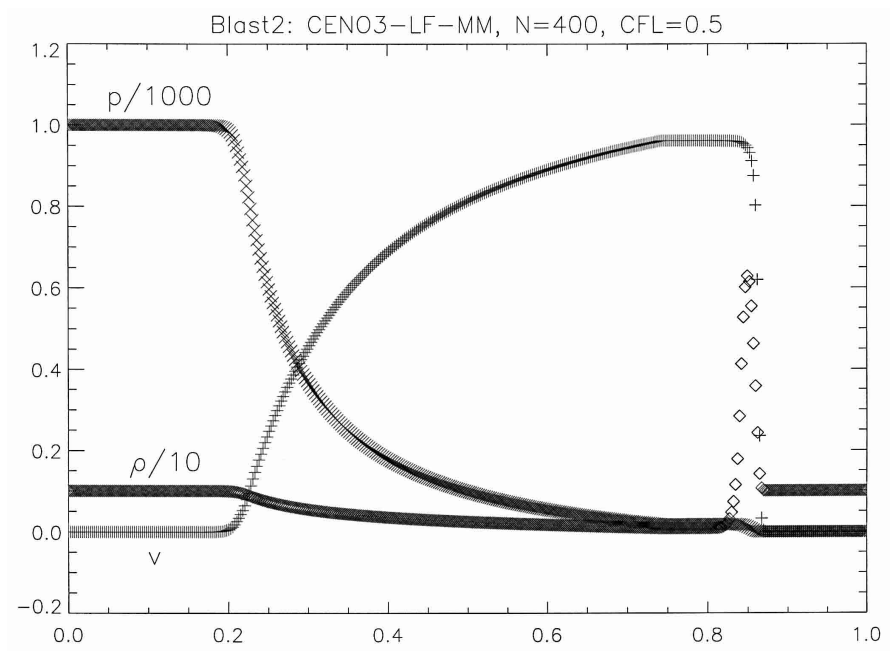

Fig. 3. The same problem and settings as in Fig. 2, except for limiter and solver. Here the most smearing case of MM limiter and global LF flux splitting with $\alpha=1$ (highest possible value for relativistic flows) is tested. Differences arise only at $x \simeq 0.2$ and $x \simeq 0.75$, where the changes of slope due to the rarefaction wave are less sharp, and at the density peak, which is lower $(\rho \simeq 6.5)$ in this case.

the base scheme CENO3-HLL-MC. Note the total absence of oscillations and the accuracy in the definition of shocks and rarefaction waves. The contact discontinuity is more smeared, due to the use of a solver that does not take into account that intermediate wave and above all to the reconstruction method, which is the same for all the quantities, thus we cannot steepen (e.g. with the superbee limiter) the contact discontinuity alone, as usually done in characteristics based schemes. In particular, the height of the density peak in Fig. 3 provides a measure of the numerical viscosity of the scheme: the exact value should be around 10.5, while here we find about 7.3 , slightly more than

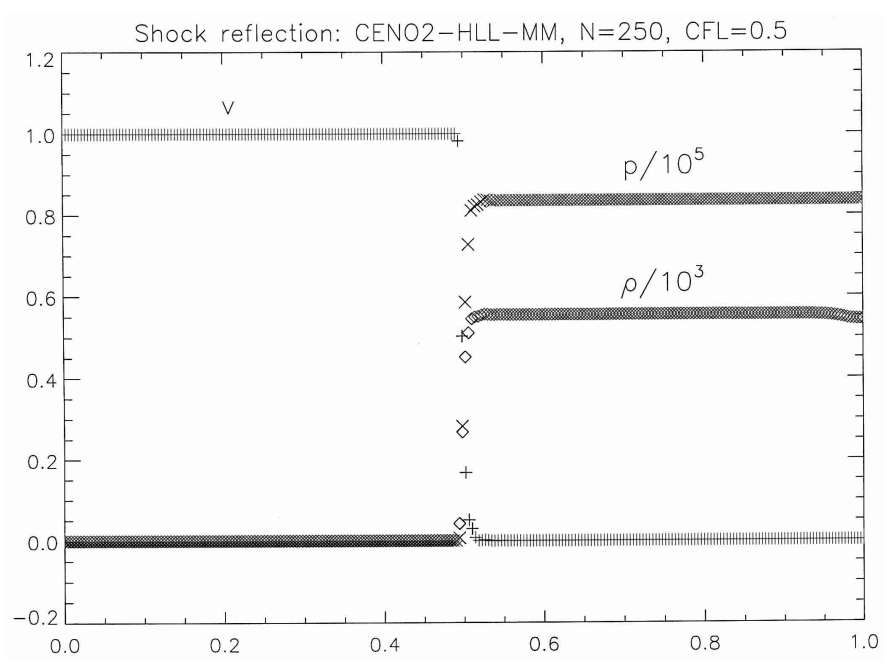

Fig. 4. The relativistic shock reflection problem for time $t=0.75$. The computed profiles of density (diamond), pressure (cross), and velocity (plus) are shown against distance. Here the second order CENO2 scheme is used with MM limiter and HLL solver. The computational grid is formed by $N=250$ equidistant cells and the Courant number is $c=0.5$. Note that the error in the density due to the wall heating phenomenon is around $2.3 \%$, to be compared with the value of $2.5 \%$ given by Marquina's scheme in its third order implementation.

what shown in Donat's paper (in their third order simulation). A smaller value, and a larger spreading, is apparent in Fig. 3, where the most smearing case is tested, that is minmod limiter and global Lax-Friedrichs solver with $\alpha=1$, corresponding to $\lambda^{ \pm} \rightarrow \pm 1$ (speed of light) in Eq. (20). However, given the extreme simplicity of the scheme, even this case should not be regarded as completely unusable.

The next example considered is the notorious relativistic shock reflection problem, where an ultrarelativistic cold wind hits a wall, a shock propagates backwards and a static region of relativistically hot gas $\left(e \gg \rho \Rightarrow c_{\mathrm{s}}^{2} \rightarrow \Gamma-1\right)$ is left behind. The numerical box is again $[0,1]$ (we use $N=250$ ) and the reflecting wall is placed at $x=1$. The physical values employed are:

Shock reflection: $(\rho, v, p)=(1,0.99999,0.01)$,

that corresponds to a Lorentz factor as high as $\gamma \simeq 223$, about the highest allowed in single precision calculations. This is a very severe test, for the high velocities involved and for the so-called wall heating problem, visible as a dip in the density profile near the reflecting wall. This is a classical numerical artifact, due basically to the implicit numerical viscosity present in every scheme. In Fig. 4 a simulation with second order accuracy (both in time and space), HLL solver and MM limiter is shown for time $t=0.75(c=0.5)$. This is the only case where the third order CENO3 has failed, giving significant postshock oscillations, that can only be reduced by lowering the Courant number or by enhancing numerical viscosity, though they never completely disappear. However, the overheating error in the density is here only $2.3 \%$, in spite of the second order of accuracy and of our simplified scheme, to be compared with the $2.5 \%$ value of Donat's paper in their third order implementation of the celebrated Marquina's scheme. 


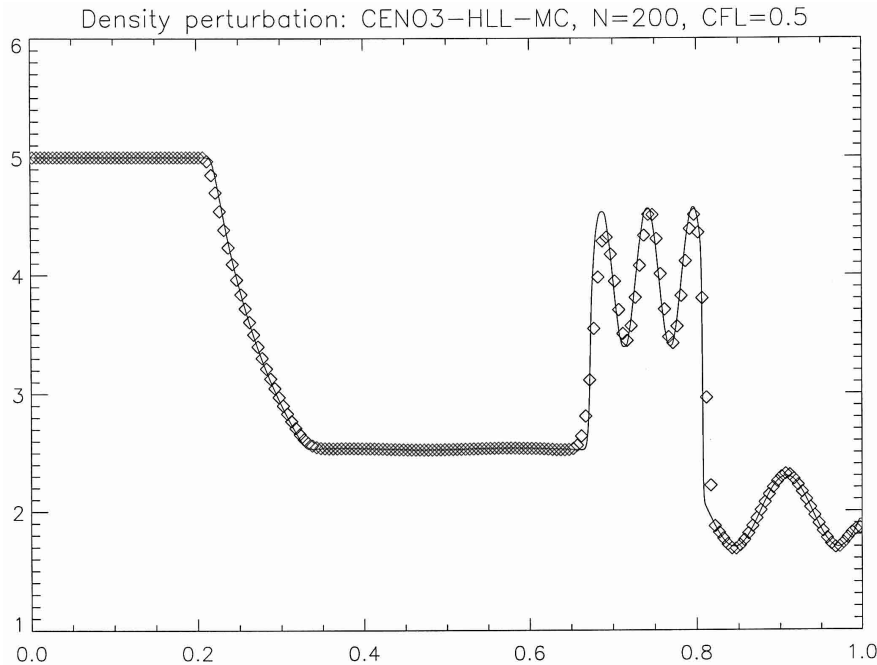

Fig. 5. The Riemann problem with sinusoidal density perturbation for time $t=0.35$. The diamonds are the results obtained with CENO3, MC limiter and HLL solver, in a simulation with $N=200$ grid points and Courant number is $c=0.5$. The solid line is the density profile that comes out from a simulation with 2000 grid points. High-order ENO schemes are particularly suited for problems involving shocks interacting with smooth wave-like structures.

As a last 1-D application we present a test proposed in the Dolezal \& Wong (1995) paper, which nicely shows the capacity of ENO-based schemes of treating accurately both discontinuous and smooth features occurring close together and at the same time. A shock tube is perturbed in its right-hand state $(0.5<x \leq 1)$ with a density sinusoidal profile:

Density perturbation: $\left\{\begin{array}{l}(\rho, v, p)^{\mathrm{L}}=(5,0,50), \\ (\rho, v, p)^{\mathrm{R}}=(2+0.3 \sin 50 x, 0,5) .\end{array}\right.$

The subsequent evolution in time shows the interaction of the blast wave with the density wave (the values are not exactly the same as in Dolezal's paper, where a nuclear equation of state is used), which enters the expanding heated region and modulates its density plateau. In Fig. 5 the extreme accuracy of our third order CENO3 scheme is shown, by comparing the resulting density profiles at time $t=0.4$ in two runs, one with just $N=200$ points (diamonds), tested against one with $N=2000$ grid points (solid line). Again, by comparing our results to those obtained by the third order characteristics based ENO scheme in Dolezal's paper, it is apparent that no significant differences arise, in spite of the much less effort involved in our scheme.

\subsection{Multidimensional tests}

Multidimensional relativistic simulations are more difficult than one-dimensional ones because the velocity components are spatially interpolated separately, possibly causing the condition $v^{2}<1$ to fail in ultrarelativistic regimes due to numerical errors in the reconstruction. For this reason in some cases we had to reduce to first order reconstruction, namely when the Lorentz factor reaches $\gamma=10$. Note that this does not mean in any sense that this threshold cannot be exceeded, but only that

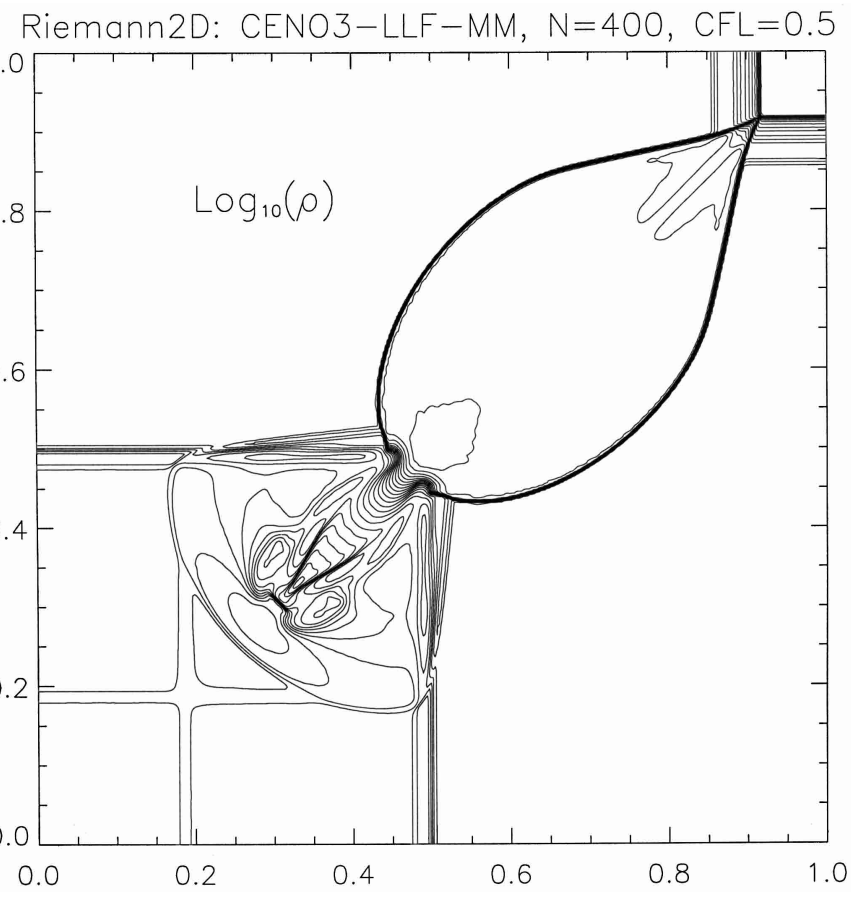

Fig. 6. The relativistic 2-D Riemann problem for time $t=0.4$. Density contours (30) in logarithmic scale are shown, for a simulation using CENO3, MM limiter and LLF solver, with $N_{x}=N_{y}=400$ grid points and Courant number is $c=0.5$.

the accuracy is lower in these regions, which usually are located at shock fronts where the order would have been lowered anyway. We have also tested reconstruction on four-velocity components, which are not bounded, but in this way the problem is just shifted to the primitive variables finding routine, thus with no improvement at all.

While it easy to test one-dimensional codes, since Riemann problems can be solved exactly through iterative algorithms, it is not so in more than one dimension, where it is rare to see really quantitative numerical scheme validations. Here we will propose a 2-D Riemann problem, 2-D and 3-D explosions, compared with the correspondent 1-D cylindrical and spherical solutions, and finally one simulation of a relativistic jet, which is not a quantitative test but it is a true astrophysical application.

The two-dimensional counterpart of shock tubes is a square domain divided in four quadrants of constant values at initial time and free evolution for $t>0$. In Lax \& Liu (1998) all the possible different configurations involving 1-D shocks, 1-D rarefactions waves and 2-D slip lines (contact discontinuities) were studied in detail. In Fig. 6 we show the output (contours of the density logarithm for time $t=0.4$ ) of a situation similar to their configuration 12, where the four boundaries defines two contact discontinuities and two 1-D shocks (symmetric with respect to the main diagonal). Here a relativistic version of this test is proposed, with the following initial settings:

Riemann 2-D: $\left\{\begin{array}{l}\left(\rho, v_{x}, v_{y}, p\right)^{\mathrm{NE}}=(0.1,0,0,0.01), \\ \left(\rho, v_{x}, v_{y}, p\right)^{\mathrm{NW}}=(0.1,0.99,0,1), \\ \left(\rho, v_{x}, v_{y}, p\right)^{\mathrm{SW}}=(0.5,0,0,1), \\ \left(\rho, v_{x}, v_{y}, p\right)^{\mathrm{SE}}=(0.1,0,0.99,1) .\end{array}\right.$ 

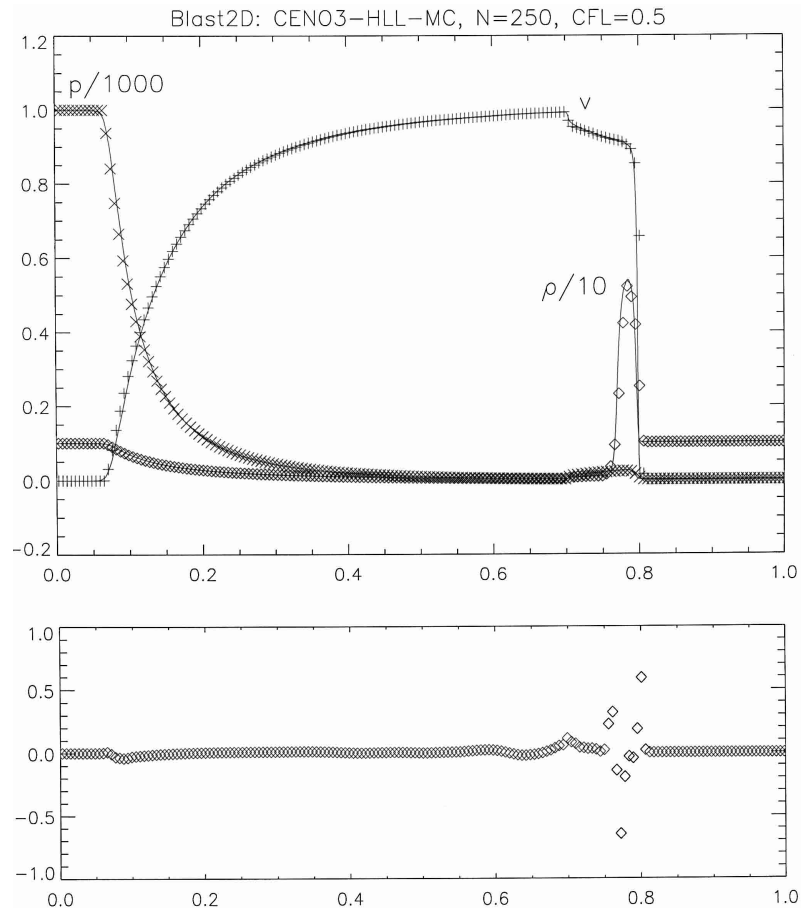

Fig. 7. The relativistic 2-D blast wave problem for time $t=0.4$. Here the simulation employed CENO3 reconstruction, MC limiter and HLL solver, with $N_{x}=N_{y}=N=250$ grid points. The domain is a Cartesian 2-D unit box $[0,1] \times[0,1]$, with initial enhanced pressure (a factor of $10^{3}$ ) in a disk sector centered at the origin with radius $r_{\max }=0.4$ (reflecting boundary conditions are assumed for $x=0$ and $y=0$ ). A radial cut (along the main diagonal) of the computed quantities are compared with those obtained through a high-resolution $(N=800)$ 1-D simulation in cylindrical coordinates (solid line), using the same parameters. For a more quantitative comparison, in the bottom panel the density relative error is plotted. Its rather large value near shocks is simply due to the naturally reduced accuracy at discontinuities and to the very small number of grid points (eight) present in the density peak.

Note that we have not taken exact 1-D shocks across the $\mathrm{N}$ and $\mathrm{E}$ interfaces, and this may be recognized by observing the evolved discontinuities in Fig. 6, converging towards the NE corner with their complete Riemann fans. In the rest of the domain the structure evolves with curved shock fronts and a complicated pattern in the SW quadrant, reminiscent of an oblique jet with bow shock and converging internal shock fronts. The lines in the SW direction with respect to the bow shock are actually due to spurious waves created at the $\mathrm{W}$ and $\mathrm{S}$ interfaces by the numerical diffusion term in the energy equation (left and right states have a jump in kinetic energy), and cannot be removed nother than by using a Roe-type solver. Note that the most diffusive case (LLF solver and MM limiter) was used in the simulation, since other cases are more unstable at the same high resolution and with the same Courant number $c=0.5$.

Figures 7 and 8 refer respectively to the 2-D cylindrically symmetric and 3-D spherically symmetric blast wave explosion problem below:

Radial blast wave: $\begin{cases}\left(\rho, v_{r}, p\right)=(1,0,1000) & r \leq 0.4, \\ \left(\rho, v_{r}, p\right)=(1,0,1) & r>0.4\end{cases}$

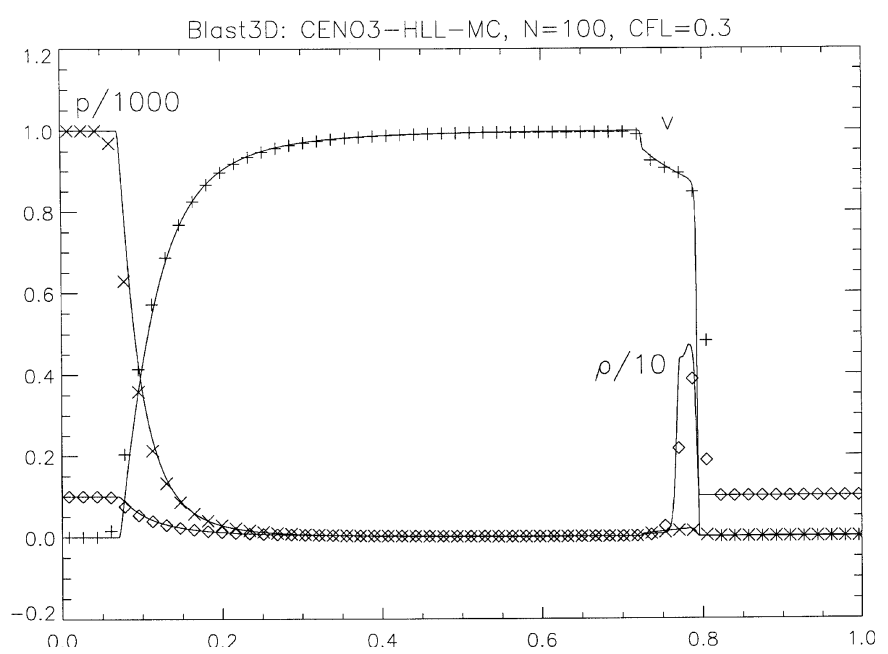

Fig. 8. The relativistic 3-D blast wave problem for time $t=0.4$. All the settings are the same as in Fig. 7, except that here $N=100$ along all directions and the Courant number is lower $(c=0.3)$. A radial cut (along the main diagonal) of the computed quantities are compared with those obtained through a high-resolution $(N=800) 1$-D simulation in spherical coordinates (solid line), using the same parameters.

where in both cases the runs have been performed in a Cartesian unit box (with reflective right hand side boundary conditions along each direction) and compared with the correspondent 1-D radial solution, obtained with $N=800$ grid points in cylindrical or spherical coordinates. The results are satisfactory, since the radial symmetry is well preserved (here the results along the diagonal are shown) and high Lorentz factors are reached without particular problems $(\gamma>25$ in the spherically symmetric case). Here the scheme used is CENO3 with HLL solver and MC limiter, with $c=0.5$ and $c=0.3$ in the 2-D and 3-D cases, respectively.

Finally, as a typical astrophysical test, we simulate the propagation of an axisymmetric jet in 2-D cylindrical coordinates $(z, r)$. Note that jet simulations are a very hard test for codes not based on characteristics decomposition, because of usually stronger numerical viscosity at shear layers. The domain is $0<r<8$ and $0<z<20$, with reflective boundary conditions on the axis $r=0$ and simple extrapolation at the other boundaries (except at $z=0$ within the jet radius, where initial values are kept constant). At $t=0$ a relativistic jet with $v_{z}=0.99$ and density 100 times less than the surroundings (but same pressure) is located at $r \leq 1$ and $z \leq 1$

Jet: $\begin{cases}\left(\rho, v_{z}, v_{r}, p\right)=(0.1,0.99,0,0.01) & r \leq 1, z \leq 1, \\ \left(\rho, v_{z}, v_{r}, p\right)=(10,0,0,0.01) & \text { outside; }\end{cases}$

density and velocity jumps are actually smoothed in order to reduce spurious transverse waves that appear due to numerical viscosity at the shear layer. The material is injected with a Lorentz factor $\gamma \simeq 7.1$, corresponding to a relativistic Mach number (e.g. Duncan \& Hughes 1994) of $\mathcal{M}=\gamma v / \gamma_{c_{\mathrm{s}}} c_{\mathrm{s}} \simeq 17.9$. The jet evolution is followed until $t=40$, as shown in Fig. 9, where density contours and gray shades in logarithmic scale are presented. The code settings are CENO3-HLL-MC with Courant coefficient $c=0.25$, while the resolution employed is $400 \times 160$, corresponding to 20 grid points per jet radius. 


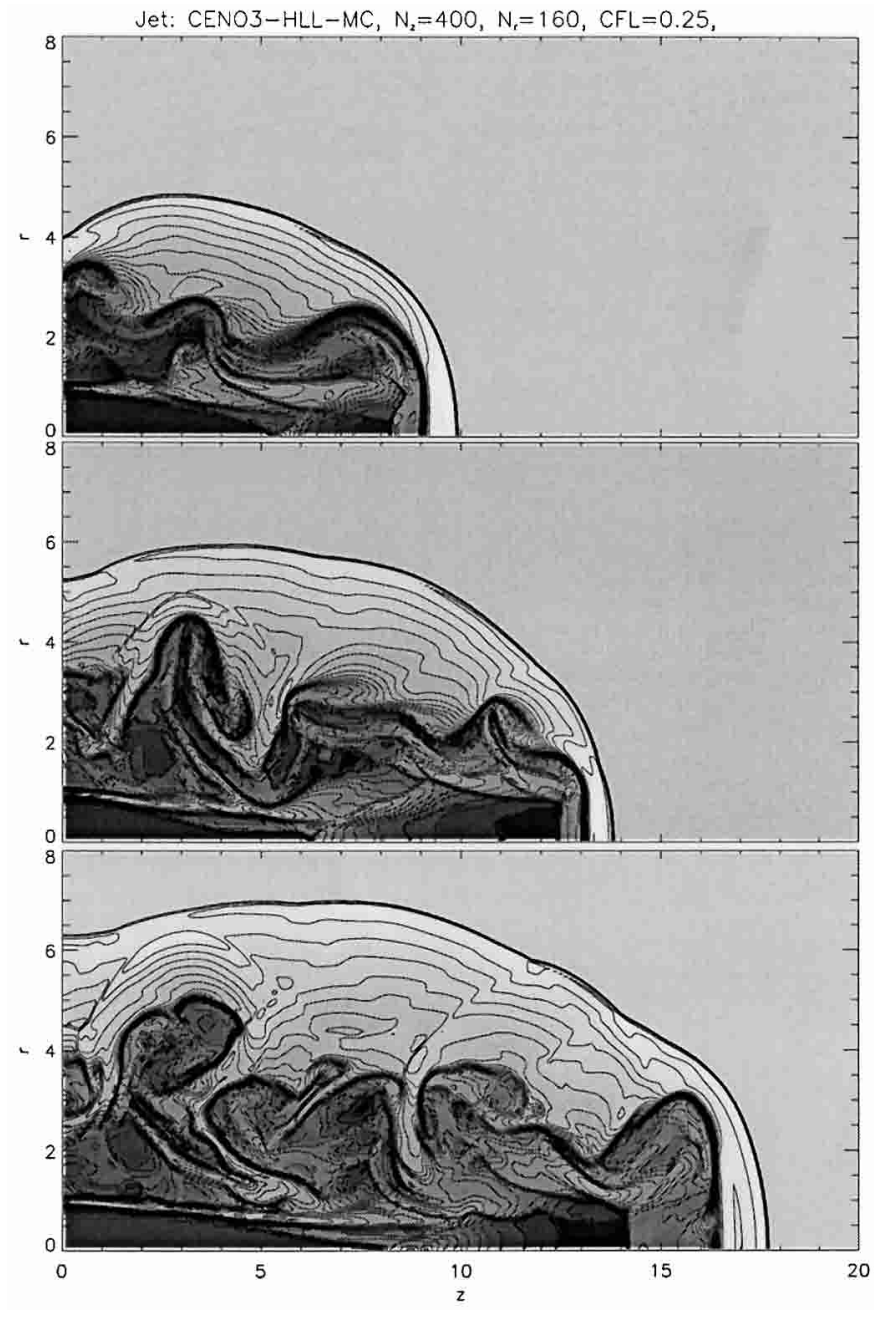

Fig. 9. The relativistic jet for times $t=20,30,40$ in 2-D cylindrical geometry (logarithmic density contours and shades are shown). The inflow speed is $v_{z}=0.99$ and the internal to external density ratio is $\eta=1 / 100$, with an overall pressure equilibrium, leading to a beam relativistic Mach number o 17.9. The jet radius is assumed as length unit, corresponding to 20 computational cells $\left(N_{z}=400\right.$ grid points in the axial direction and $N_{r}=160$ points in the radial direction). CENO3 reconstruction, HLL solver and MC limiter have been used in the simulation, with Courant number $c=0.25$.

Note that the smearing of contact discontinuities, unavoidable in methods not based on characteristics decomposition, is actually small and vortices due to Kelvin-Helmoltz instabilities are nicely defined, as well as the external bow shock, the internal Mach disk and other shocks reflected off the axis. Moreover, notice the absence of the so-called carbuncle problem, usually manifesting as an extended nose in the front of the jet on the axis (e.g. Quirk 1994).

\section{Conclusions}

The central shock-capturing scheme of Londrillo \& Del Zanna (2000) is extended to the relativistic case, here for ideal RHD flows and to RMHD in the next paper of the series. Compared to other schemes proposed for relativistic astrophysical problems over the last decade in the literature, our method is extremely simple and efficient, since no eigenvector decomposition and Riemann solvers are involved. In spite of this, due to the high order accuracy achieved in smooth regions and to TVD limiting near discontinuities, provided by the CENO reconstructions employed, our results compete with those obtained by more sophisticated (but less computationally efficient) codes, even in 2-D and 3-D highly relativistic test problems. Different geometries (cylindrical and spherical coordinates) and boundary conditions have also been tested. All the numerical experiments have been run on a simple PC-Linux machine, even the most demanding 3-D test with one million grid points, in order to demonstrate the efficiency of our code. We believe that our simple scheme can be successfully employed in many relativistic simulations of astrophysical interest, either in the fluid case and above all in the most demanding magnetic case, which will be the subject of a forthcoming paper.

Acknowledgements. The authors thank P. Londrillo and R. Bandiera for valuable discussions. This work has been partly supported by the Italian Ministry for University and Research (MIUR) under grants Cofin2000-02-27 and Cofin2001-02-10.

\section{Appendix A: Convex ENO interpolation routines}

Given a function $v(x)$ with grid point values $\left\{v_{i}\right\}$, for $i=$ $1, \ldots, N$, let us see how non-oscillatory reconstructions $(\operatorname{Rec})$ and second derivatives (Der) are defined in our scheme. These procedures are based on the original CENO reconstruction from Liu \& Osher (1998) and they have been described also in LD (we repeat them here for completeness).

In the range $x_{i-1 / 2} \leq x_{i} \leq x_{i+1 / 2}$, we first consider the two linear polynomials $L_{i}(x)$, based on the stencils $\left[x_{i-1}, x_{i}\right]$ or $\left[x_{i}, x_{i+1}\right]$, and we choose their convex combination which is closest to $v_{i}$, that happens to coincide with the TVD limited reconstruction:

$\tilde{L}_{i}(x)=v_{i}+v_{i}^{\prime}\left(\frac{x-x_{i}}{\Delta x}\right)$

Here the non-oscillatory undivided first derivative $v_{i}^{\prime}$ is given by the minmod function:

$v_{i}^{\prime}=\operatorname{mm}\left[\Delta_{-} v_{i}, \Delta_{+} v_{i}\right]$

and $\Delta_{ \pm} v_{i}= \pm\left(v_{i \pm 1} \pm 1\right)$. Thus, to second order of accuracy the CENO technique simply reduces to the usual TVD monotone slope limiting. Although not in the philosophy of the original CENO approach, we have tested other limiters. The most compressive ones, like superbee are too oscillatory in our component-wise framework, while a good compromise between sharpening and stability appears to be provided by the so-called monotonized centered (TVD for $1 \leq \theta \leq 2$ ):

$v_{i}^{\prime}=\operatorname{mm}\left[\theta \Delta_{-} v_{i}, \Delta_{0} v_{i}, \theta \Delta_{+} v_{i}\right]$,

where $\Delta_{0} v_{i}=\left(\Delta_{+} v_{i}+\Delta_{-} v_{i}\right) / 2=\left(v_{i+1}-v_{i-1}\right) / 2$ and $\theta=2$ in order to bias towards central interpolation (since twice the one-sided derivatives might be chosen near discontinuities, the Courant 
coefficient should be lowered correspondingly for overall stability). In both relations the function $\mathrm{mm}$ is defined as

$\operatorname{mm}\left(a_{1}, a_{2}, \ldots\right)= \begin{cases}\min _{j}\left\{a_{j}\right\} & a_{j} \geq 0 \forall j, \\ \max _{j}\left\{a_{j}\right\} & a_{j} \leq 0 \forall j \\ 0 & \text { otherwise }\end{cases}$

To achieve an accuracy of third order in smooth regions, we need to calculate the three quadratic polynomials $(k=$ $-1,0,+1, j=i+k)$ :

$Q_{i}^{k}(x)=v_{j}+\Delta_{0} v_{j}\left(\frac{x-x_{j}}{\Delta x}\right)+\frac{1}{2} \Delta_{0}^{2} v_{j}\left(\frac{x-x_{j}}{\Delta x}\right)^{2}$

where $\Delta_{0}^{2} v_{j}=\Delta_{+} \Delta_{-} v_{j}=\Delta_{-} \Delta_{+} v_{j}=v_{j+1}-2 v_{j}+v_{j-1}$, together with the corresponding weighted differences

$d_{i}^{k}=\alpha^{k}\left(Q_{i}^{k}-\tilde{L}_{i}\right)$.

Then, we take again their convex combination which is closest to $\tilde{L}_{i}$ at a given point $x$ (this procedure is the generalization of minmod limiting to higher orders) and finally, in smooth regions where all $d_{i}^{k}$ have the same sign, we take

$\tilde{Q}_{i}=Q_{i}^{k_{0}},\left|d_{i}^{k_{0}}\right|=\min _{k}\left(\left|d_{i}^{k}\right|\right)$,

otherwise we exit from the interpolation routine with $\tilde{Q}_{i}=\tilde{L}_{i}$. As for the coefficient $\theta$ in the limiter of the lower order, the weights $\alpha^{k}$ are chosen in order to bias towards central interpolation, and following the original recipe for component-wise CENO schemes we take $\alpha^{ \pm 1}=1$ and $\alpha^{0}=0.7$. In our Rec routine, the left and right states are calculated at the same time and the output is finally

$v_{i+1 / 2}^{\mathrm{L}}=\tilde{Q}_{i}\left(x_{i+1 / 2}\right), v_{i-1 / 2}^{\mathrm{R}}=\tilde{Q}_{i}\left(x_{i-1 / 2}\right)$.

The other CENO routine used in our scheme is Der, which allows one to calculate non-oscillatory second derivatives $\bar{v}_{i}^{\prime \prime}$, needed to transform cell averages into point values, calculated at the same point:

$\left\{\bar{v}_{i}\right\} \longrightarrow\left\{v_{i}\right\}, \quad v_{i}=\bar{v}_{i}-\frac{1}{24} \bar{v}_{i}^{\prime \prime}$.

In this case, the above CENO selection criterion applies with null linear polynomials, $\tilde{L}_{i}=0$, and quadratic ones given by $(k=-1,0,+1, j=i+k)$ :

$Q_{i}^{k}=\Delta_{0}^{2} \bar{v}_{j} \equiv \bar{v}_{j+1}-2 \bar{v}_{j}+\bar{v}_{j-1}$,

then finally $\bar{v}_{i}^{\prime \prime}=\tilde{Q}_{i}$ (which is zero, so $\bar{v}_{i} \equiv v_{i}$ as for second order approximation, in the case different signs of the $Q_{i}^{k}$ terms).

\section{References}

Aloy, M. A., Ibañez, J. M., Marti, J. M., \& Müller, E. 1999, ApJS, 122,151

Anile, M. 1989, Relativistic Fluids and Magneto-Fluids (Cambridge University Press, Cambridge)

Balsara, D. S. 1994, J. Comput. Phys., 114, 287

Balsara, D. S. 2001, ApJS, 132, 83

Begelman, M. C., Blandford, R. D., \& Rees, M. J. 1984, Rev. Mod. Phys., 56, 255

Bucciantini, N., \& Bandiera, R. 2001, A\&A, 375, 1032

Dolezal, A., \& Wong, S. S. M. 1995, J. Comput. Phys., 120, 266

Donat, R., Font, J. A., Ibañez, J. M., \& Marquina, A. 1998, J. Comput. Phys., 146, 58

Duncan, G. C., \& Hughes, P. A. 1994, ApJ, 436, L119

Eulderink, F., \& Mellema, G. 1994, A\&A, 284, 654

Falle, S. A. E. G., \& Komissarov, S. S. 1996, MNRAS, 278, 586

Ferrari, A. 1998, ARA\&A, 36, 539

Font, J. A., Ibañez, J. M., Marquina, A., \& Marti, J. M. 1994, A\&A, 282,304

Harten, A., Engquist, B., Osher., S., \& Chakravarthy, S. 1987, J. Comput. Phys., 71, 231

Jiang, G., Shu, C.-W. 1996, J. Comput. Phys., 126, 202

Kennel, C. F., \& Coroniti, F. V. 1984, ApJ, 283, 694

Kobayashi, S., Piran, T., \& Sari, R. 1999, ApJ, 513, 669

Komissarov, S. S. 1999, MNRAS, 303, 343

Kurganov, A., Noelle, S., \& Petrova, G. 2001, SIAM J. Sci. Comput., 23, 707

Landau, L. D., \& Lifshitz, E. M. 1959, Fluid Mechanics (Pergamon Press)

Lax, P. D., \& Liu, X.-D. 1998, SIAM J. Sci. Comput., 19, 319

Liu, X.-D., \& Osher, S. 1998, J. Comput. Phys., 142, 304

Londrillo, P., \& Del Zanna, L. 2000, ApJ, 530, 508 (LD)

Marquina, A., Marti, J. M., Ibañez, J. M., Miralles, J. A., \& Donat, R. 1992, A\&A, 258, 566

Meszaros, P., \& Rees, M. J. 1992, MNRAS, 258, 41

Michel, F. C., \& Li, H. 1999, Phys. Rep., 318, 227

Mirabel, I. F., \& Rodriguez, L. F. 1999, ARA\&A, 37, 409

Nessyahu, H., \& Tadmor, E. 1990, J. Comput. Phys., 87, 408

Piran, T. 1999, Phys. Rep., 314, 575

Quirk, J. 1994, Int. J. Numer. Methods Fluids, 18, 555

Rees, M. J. 1967, MNRAS, 137, 429

Rees, M. J., \& Gunn, J. E. 1974, MNRAS, 167, 1

Schneider, V., Katscher, U., Rischke, D. H., et al. 1993, J. Comput. Phys., 105, 92

Shu, C.-W. 1997, ICASE Rep. 97-65, NASA Langley Research Center, VA

Shu, C.-W., \& Osher, S. 1988, J. Comput. Phys., 77, 439

Shu, C.-W., \& Osher, S. 1989, J. Comput. Phys., 83, 32 